\title{
Extended Source Gamma-Ray Emission from WIMP Annihilation in the Sagittarius Dwarf Elliptical Galaxy
}

\author{
VIDYA VASU-DEVAN \\ Columbia University \\ Office of Science, Science Undergraduate Laboratory Internship (SUli) \\ Stanford Linear AcCelerator Center \\ Menlo Park, CA 94025
}

August 20, 2005

Prepared in partial fulfillment of the requirements of the Office of Science, Department of Energy's Science Undergraduate Laboratory Internship under the direction of DR. LARRY WAI in Group K of the Kavli Institute of Particle Astrophysics and Cosmology (KIPAC) at the Stanford Linear Accelerator Center (SLAC).

Participant:

Signature

Research Advisor:

Signature 


\section{Contents}

1 INTRODUCTION $\quad 3$

2 INSTRUMENT AND OBSERVATIONS

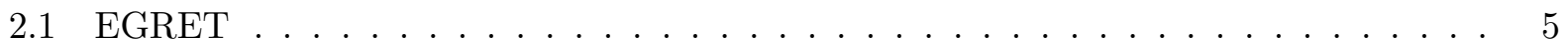

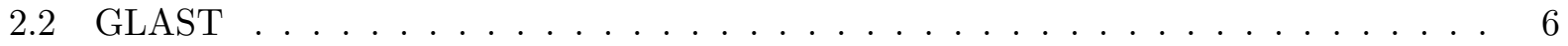

$\begin{array}{lll}3 & \text { METHOD OF ANALYSIS } & 7\end{array}$

3.1 Determination of the Background Model . . . . . . . . . . . . . . 7

3.1 Background Origination . . . . . . . . . . . . . 7

3.1.2 Method of Extrapolating Background From Data . . . . . . . . . . . . 8

3.1.3 Combining Viewing Periods . . . . . . . . . . . . . . . . 8

3.1.4 Monte Carlo Generation of Point Sources . . . . . . . . . . . . . . . . . . 8

3.2 Addition and Application of Models . . . . . . . . . . . . . . . . . . . 10

3.2 .1 Point Source Model . . . . . . . . . . . . . . . . . . . . . . 10

3.2.2 Extended Source Model . . . . . . . . . . . . . . . . . . . . 11

3.2.3 Variations of Extended Source Model . . . . . . . . . . . . . . . . 12

3.3 Hypothesis Testing . . . . . . . . . . . . . . . . . . . 12

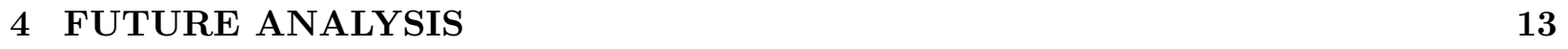

4.1 Expansion of Analysis to GLAST . . . . . . . . . . . . . . . . 13

4.2 Extension to Different Energy Range . . . . . . . . . . . . . . . . . . . . 13

5 RESULTS AND CONCLUSIONS 14

6 ACKNOWLEDGMENTS $\quad 15$

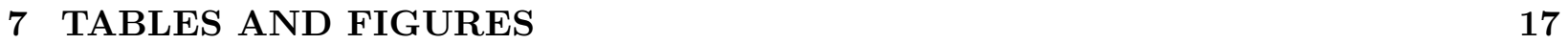




\begin{abstract}
Extended Source Gamma-Ray Emission from WIMP Annihilation in the Sagittarius Dwarf Elliptical Galaxy. VIDYA VASU-DEVAN (Columbia University, New York, NY 10027-8361) LARRY WAI (Stanford Linear Accelerator Center, Menlo Park, CA 94025).
\end{abstract}

The proximity of the dark matter dominated Sagittarius Dwarf Elliptical Galaxy (position $(l, b)$ $\left.=5.6^{\circ},-14^{\circ}\right)$ allows it to act as an ideal laboratory for the exploration of extended gamma-ray emission from Weakly Interacting Massive Particle (WIMP) annihilation processes in a dark matter-dominated system. Since the matter in our universe is predominantly dark, exploring such processes as WIMP annihilation will lead to a better understanding of cosmology. In order to study this gamma-ray emission, a model for the diffuse background gamma-radiation in the dwarf galaxy's region is extracted from the Energetic Gamma Ray Experiment Telescope (EGRET) data. After validating this model and comparing it to the EGRET diffuse model, the background model is added to effective bleeding-contamination from external point sources and multiple models for the signal-above-background emission. Various models of this emission are tested: a)no source located in region, b)unidentified point source 3EG J1847-3219 from the Third EGRET Catalog responsible for the emission and c)extended emission resulting from WIMP annihilation responsible for the signal above background. These models are created through the employment of Monte Carlo simulation methods, utilizing the response functions of the EGRET instrument to simulate the point spread function, energy dispersion and effects of variable effective area depending on angle of incidence. Energy spectra for point sources are generated from the best predictions of spectral indices listed in the Third EGRET Catalog and the spectrum for the extended dark matter source is generated from Pythia high energy annihilation simulations. Hypothesis testing is conducted to assess the goodness-of-fit of these models to the data taken by EGRET. Additionally, we hope to expand our analysis by employing the response functions of the imminent Gamma Ray Large Area Space Telescope (GLAST) to our models. This extension should highlight the sensitivity disparities between GLAST and EGRET and show GLAST's potential enhancement of this analysis. This process will allow for forecasting of extended WIMP annihilation emission signatures for the GLAST detector.

\title{
1 INTRODUCTION
}

Current understanding of the universe leads to the prediction that the universe is at critical density (i.e. has a flat geometry) with baryonic matter accounting for approximately $4 \%$ of this density, dark matter responsible for approximately $23 \%$ and the mysterious dark energy accounting for approximately $73 \%$. Thus, as the matter which inhabits the universe is predominantly non-baryonic 
(i.e. dark), pinpointing and exploring the elusive nature of dark matter is of the utmost importance in understanding the composition, dynamic systems and origin and development of our universe. Dark matter is largely responsible for the gravitational binding of large-scale structures such as galaxies and clusters of galaxies. Though many theories suggest plausible models for the nature of dark matter, including its interaction processes and characteristics, the actual constituent particles have not been identified with any level of certainty. Weakly-Interacting Massive Particles (WIMPs) are likely candidates for cold dark matter - or dark matter particles, which were not moving at relativistic speeds during the formation of galaxies. This naming device reflects the fact that these particles do not exhibit any electromagnetic or strong interaction and only interacts with baryonic matter through gravitation. The originally low speeds of cold dark matter allow for eventual clumping into structures. For a universe with critical density, the cross section of interaction among WIMPs is on the electroweak scale [1]. In order to compensate for problems with the Standard Model, supersymmetric theory postulates the neutralino, the lightest supersymmetric partner to bosons like photons, as a particle which interacts on the electroweak scale. Thus, the neutralino is a likely candidate for the Weakly-Interacting Massive Particle (WIMP). Dark matter can be both directly and indirectly detected in a variety of experimental methods. This paper will focus on the indirect detection of WIMP annihilation processes through the detection of astrophysical gamma-rays.

The fortunate proximity of many galactic satellite dwarf galaxies provides favorable targets for exploring galactic processes. The Sagittarius Dwarf Elliptical Galaxy (SagDEG) is particularly interesting since its lack of significant disruption over the course of its $\geq 10$ orbits around the Milky Way suggests that the galactic baryonic matter is enveloped by a dark matter halo. Though the galactic center also possesses a great density of dark matter, the abundance of other varied gamma-ray emitting astrophysical processes creates a region so complex and polluted, these annihilation processes are extremely difficult to isolate. Thus the location of the dark matter dominated SagDEG, which orbits the center of our galaxy and is situated off of the galactic plane, provides a more unadulterated view of the dynamics of dense clumps of dark matter. N-body simulations predict that particles, which only experience gravitational interaction, clump into dense structures [2]. Since the WIMP annihilation cross-section is proportional to the square of the WIMP density, regions like SagDEG are ideal for exploring WIMP annihilation. WIMP annihilations produce 
a quark/anti-quark final state, which fragments into hadrons. We will focus on the annihilation process which leads to the production of $\pi^{0}, \pi^{-}$and $\pi^{+}$particles. The $\pi^{0}$ particles subsequently decay into a pair of gamma-rays while the charged $\pi$ particles decay into neutrinos and leptons, which produce gamma radiation through inverse Compton scattering off of starlight as well as synchrotron radiation [3]. In this paper, we will focus on only the gamma-radiation resulting from the $\pi^{0}$ decays. This radiation is expected to have an extended signature due to the SagDEG's proximity and composition.

This paper will explore the application of a model of an extended source of WIMP annihilation in contrast to other hypotheses for the emission above background originating in the region of SagDEG. These models will be compared with data taken by the Energetic Gamma Ray Experiment Telescope (EGRET). In order to complete this analysis, we must model the diffuse gamma-ray emission background. We choose to approach this challenge by extracting a background model from the data itself. We then look at the background model and external point source contamination coupled with our models and compare with raw EGRET data. We hope to explore the signatures of extended emission, which have not been explored for the SagDEG system to date.

Additionally, we plan to conduct the same analysis for the Gamma Ray Large Area Space Telescope (GLAST). We will extrapolate the sensitivity of GLAST to such models, forecasting the signatures of dark matter annihilation which GLAST should expect to see and the potential amelioration to our analysis GLAST will allow.

\section{INSTRUMENT AND OBSERVATIONS}

\section{$2.1 \quad$ EGRET}

EGRET, located on the Compton Gamma Ray Observatory (CGRO), was a space-based instrument designed to detect gamma-rays between $20 \mathrm{MeV}$ and $30 \mathrm{GeV}$. The $2.25 \mathrm{~m}$ x $1.65 \mathrm{~m}$ detector was composed of a spark chamber tracking system, which reconstructed the direction of gamma-rays through the monitoring of pair-production in the chamber, and a NaI calorimeter, which reconstructed the energy of incoming photons. The entire detector was enclosed in a plastic scintillator anticoincidence dome, which flagged charged particles to avoid false identification of gamma-rays. Certain EGRET response functions must be corrected for when using raw EGRET counts data. First, the 
effective area of EGRET was approximately $1500 \mathrm{~cm}^{2}$ and fell off completely at $40^{\circ}$ off-axis (See FIGURE 1). EGRET's point spread function could be well approximated as an energy-dependent Gaussian function. Additionally, energy dispersion has to be taken into account. EGRET operated through localized pointing for 2 week periods (a.k.a. Viewing Periods). Due to the orbit of CGRO, the Earth would obstruct EGRET's field of view to various extents over the 2 week viewing periods, thus telescope modes need to be taken into account when using EGRET data, specifically when attempting to simulate data for a specific viewing period.

EGRET's scientific objectives included the search for gamma-ray emitting point sources and the identification of locations, energy spectra and variability. Additionally, EGRET aimed to probe supernovae remnants and investigate their cosmic ray acceleration processes, to explore the galactic and extragalactic diffuse background and to search for extragalactic high energy sources. EGRET succeeded in locating and specifying the energy distributions of 271 point sources. Among these point sources are pulsars, galaxies, solar flares, AGNs and 170 unidentified astrophysical objects [4]. However, Third EGRET Catalog only positively identified one extended source, which required a highly specialized analysis [5]. Additionally, EGRET data was used to create the first sensitive map of diffuse emission.

\subsection{GLAST}

GLAST, which will be launched in 2007, is the next generation space-based gamma-ray detector. It is designed to detect gamma-rays in the approximate range of $10 \mathrm{MeV}$ to $300 \mathrm{GeV}$. The GLAST Large Area Telescope (LAT) will have approximately 50 times the sensitivity and twice the field of view of EGRET. The effective area as a function of angle off-axis is plotted in FIGURE 1. LAT contains 16 towers of silicon detectors, which track the pair-production electrons and positrons. The CsI calorimeter employs scintillation and photoelectric conversion to determine the energy of the detected photons. Like EGRET, GLAST is entirely surrounded by an anticoincidence shield, which discriminates between actual gamma-ray events and the charged particle background. Also like EGRET, future GLAST raw data will need to be corrected for the effective area, energy dispersion and point spread function. However, GLAST will be a scanning mission and thus will not have viewing periods. As a result, the complex rotation and movement of the instrument, while in orbit, will need to be taken into account. 
GLAST aims to investigate the acceleration processes in such high energy emitting astrophysical sources as supernovae remnants, AGN and pulsars. Additionally, GLAST aims to improve upon the EGRET model of diffuse emission and extend the catalog of gamma-ray point sources. GLAST also plans on using the detection of gamma-rays to probe the interaction of dark matter. Due to increased sensitivity, GLAST will be able to explore extended emission of such interactions in great detail, hopefully uncovering much of the mystery of the dark matter, which dominates our universe.

\section{METHOD OF ANALYSIS}

\subsection{Determination of the Background Model}

\subsubsection{Background Origination}

In order to carry out this analysis, an appropriate model for the background diffuse emission has to be created. The galactic diffuse emission results primarily from the interaction of cosmic rays with the interstellar medium. The cosmic ray electrons produce gamma-rays through bremsstrahlung radiation while the protons are involved in nucleon-nucleon processes. Additionally, a contribution arises from these charged particles producing gamma-rays through inverse Compton scattering off of Cosmic Microwave Background (CMB) photons and starlight. The heavily gamma-ray emitting galactic plane does not allow for the demarcation and identification of possible point sources located along the galactic plane. These unresolved sources also contribute to the galactic diffuse emission [6]. There is also an indication that cosmic rays interact with molecular gas clouds, which are prevalent in our galaxy [7]. These gas clouds (primarily molecular hydrogen) are a likely candidate for a portion of the composition of the baryonic dark matter. Cosmic rays interact with these gas clouds producing $\pi^{0}$ particles. These $\pi^{0}$ s subsequently decay into two gamma-rays. The preceding components of the galactic diffuse emission are represented in the following equation, in which q represents a gamma-ray source function, $\mathrm{N}_{H I}$ and $\mathrm{N}_{H I I}$ represent neutral and ionized hydrogen, respectively, and $\mathrm{I}_{I C}$ and $\mathrm{I}_{E G}$ represent the inverse Compton and extragalactic contributions [7]:

$$
I=I_{\text {clouds }}+\left(q_{p p \rightarrow \pi^{0}}+q_{\text {brems }}\right)\left(N_{H I}+N_{H I I}\right)+I_{I C}+I_{E G}
$$




\subsubsection{Method of Extrapolating Background From Data}

We choose to extract our background model directly from the EGRET data. Since the source region is located at a significant distance from the galactic plane $(b=-14)$, it is possible to well-model the background originating in the highly-emitting galactic plane in the area around the source region. Thus, it is possible to take regions located on either side of the source region in longitude and then interpolate a background model across the source region.

The following region fully contains the source, SagDEG: $0^{\circ}<l<8^{\circ}$ and $-18^{\circ}<b<-10^{\circ}$. We select the following regions as the two surrounding background regions: 1) $-8^{\circ}<l<0^{\circ}$ and $-18^{\circ}$ $<b<-10^{\circ}$ and 2$) 8^{\circ}<l<16^{\circ}$ and $-18^{\circ}<b<-10^{\circ}$. We create a step function for $2^{\circ}$ latitude steps, which slopes between the average value of counts in region 1 and the average value in region 2. Thus the interpolated background model consists of four independent background slopes across the $8^{\circ}$ in longitude (one for each step down in latitude). Stepping down over latitude is necessary since the number of counts decreases as the latitude value moves further away from the galactic plane.

In order to extract this step function, we must remove both the EGRET point sources located in these surrounding regions and the point sources located outside of these regions, which bleed in due to the point spread function of the detector.

\subsubsection{Combining Viewing Periods}

In order to conduct an analysis, which maximizes EGRET sensitivity, we choose to merge data from multiple viewing periods, which provides much higher statistics. After looking at the Third EGRET Catalog, we selected the viewing periods which have flagged $\geq 30$ counts for unidentified source 3EG J1847-3219 which EGRET located at $(l, b)=3.21^{\circ},-13.37^{\circ}$, which corresponds to signal generated from our source region. The viewing periods and their locations are located in TABLE 1.

\subsubsection{Monte Carlo Generation of Point Sources}

To remove the bleeding sources, we run a Monte Carlo simulation for each point source to recreate the photon events seen by the detector in each viewing period. The energies of the photons originating from this source must be distributed from the power-law spectrum associated with the 
source. These power-law indices and their associated errors are contained in the Third EGRET Catalog. Additionally, the counts and locations need to be "spread" using the appropriate EGRET response functions (point spread function, energy dispersion function, and effective area correction). Additionally, to accurately recreate the data from each viewing period, we extract the amount of live time spent in each telescope mode and generate the photon events accordingly. Thus, 10,000 photon events per viewing period are Monte Carlo generated from the source spectrum, with the correct proportion coming from each telescope mode. We spread these counts' locations and energies using the response functions and smear in the effective area function to account for the falling off effect for photons detected off-axis. The parameters associated with each of the point sources, which bled into the background regions, are contained in TABLE 2.

Sources 3EG J1800-3955 and 3EG J1911-2000 have been identified as AGNs, while the other seven sources are unidentified. As forementioned, the unidentified 3EG J1847-3219, located at $l$ $=3.21, b=-13.37$, lies inside the source region. Thus the bleeding effects of this source into the outside background regions also needs to be taken into account. Plots of the true power-law energy spectrum, dispersed energy spectrum and point spread function for an example point source, highlighting the effects of the detector on the data, are shown in Figure 2, Figure 3, \& Figure 4.

Once the simulation has been completed, the events that fall into the viewing period field and energy range $(100 \mathrm{MeV}-10 \mathrm{GeV})$ must be calculated. That total number is then scaled down to the total number of source events detected by EGRET for each viewing period. For many sources, the Third EGRET Catalog only lists upper limits for counts detected from the source in the viewing period, thus we must calculate the best normalization factor from the source flux value. EGRET lists flux values associated with each source, corresponding to the average value over the first four phases of data. We take this value and convolve it with the viewing period exposure at the location of the point source to determine the best number of events EGRET could attribute to the source. These values are our scaling factors for the Monte Carlo counts. In the case that this value exceeds the upper limit of viewing period counts listed in the Third EGRET Catalog, we choose to use the upper limit. Similarly, for variable sources like AGNs, we choose to use listed absolute count values rather than the flux-calculated values since the average flux value is inaccurate. Once we normalize our simulated sources, we add the photons detected in each $.5^{\circ} \times .5^{\circ}$ bin from all of the bleeding 
point sources and viewing periods.

It is now possible to model the background. In order to do this, we take the merged raw EGRET counts data from the five viewing periods and subtract this totaled point source contribution. The resulting counts are considered the background values for each bin. It is at this point that we calculate the average number of counts for $2^{\circ}$ latitude slices in each background region and then interpolate a crooked step function across the source region. A three-dimensional plot of the background model is located in Figure 5.

\subsection{Addition and Application of Models}

The background model is now added to the external point source contamination in the source region and hypothesis models. In order to determine the likely source of the signal above background, we consider three separate hypotheses. First, no source lies in signal region; only our background model lies in the region. Second, the EGRET point source 3EG J1847-3219 is responsible for all emission from this region. And third, the extended WIMP annihilation source in SagDEG is responsible for the signal in this region.

The first model involves only the background model obtained from the data as delineated above.

\subsubsection{Point Source Model}

First, we hypothesize that a point source lying in the source region corresponds to the EGRET data. Since EGRET flagged 3EG J1847-3219 as a point source located in the signal region, we used the catalog's precise location and the EGRET power-law index to Monte Carlo generate a model. In order to correct for the detector effects we follow the same process as previously described for dealing with external point source contamination. Similarly, we follow the same process to scale the Monte Carlo counts down to an appropriate value to compare with the EGRET data.

Once we scale this model we generate a plot, which shows the raw EGRET counts and the model to facilitate easy comparison. We choose to show our data and model in longitude and latitude profiles. Taking the longitude summed into one-degree wide bins, we sum all of the data over the eight-degree latitude source region. The same process is followed for the latitude profile. We then plot the raw EGRET counts with statistical errors(black), the background value associated with each bin (red), the background model (the average of the values for each bin) (blue), the background 
model with external point source contamination within the source region (green), and finally the point source model: the background model with the external point source contamination and the model for the point source signal (cyan). Figure 6 \& Figure 7 show plots of the longitude and latitude counts profiles associated with the signal region for this point source.

Additionally, we create an alternate model for the point source utilizing a different normalization process. Since we are using a different background model than the EGRET diffuse model, the normalization counts value will differ. Thus, we choose to conduct the analysis again by choosing a normalization value, which corresponds to the minimum $\chi^{2}$ value. This requires looping through various normalization (EGRET total count values for the point source) and calculating the corre-

sponding $\chi^{2}$ values. FiguRE 8 shows the normalization values and their corresponding $\chi^{2}$ values. We find that 210 is the optimal normalization value and plot the longitude and latitude profiles for this model in Figure 9 \& Figure 10.

\subsubsection{Extended Source Model}

Our alternative hypothesis states that extended emission generated by WIMP annihilation in the dark matter dominated SagDEG region accounts for the signal seen in our source region. In order to create a model for such emission, we need the WIMP annihilation cross section at non-relativistic velocities and the spectrum of annihilation products [3]. WIMP annihilation can be approximated as a $q / \bar{q}$ final state. Using Pythia, a program which simulates final quark state decays, we simulate $\mathrm{b} / \overline{\mathrm{b}}$ final states (since the WIMP is expected to have mass in the $\mathrm{GeV}-\mathrm{TeV}$ range, we chose the most massive quark final state) [8]. These final states hadronize into $\pi^{0}, \pi^{-}$and $\pi^{+}$particles in roughly even branching fractions. We ignore the charged pions decay into leptons (which produce gamma rays through inverse Compton scattering off of present photons) and focus on the direct $\pi^{0}$ decays. Thus, for the purposes of our model, we consider only the $\pi^{0} \mathrm{~s}$ and their subsequent decay into gamma-rays. Using Pythia, we generated the energy spectrum, seen in FigURE 11, for WIMP annihilation. This figure shows spectra for WIMP masses of $10 \mathrm{GeV}, 100 \mathrm{GeV}$ and $1000 \mathrm{GeV}$.

Extrapolating broken power-laws from the $100 \mathrm{GeV}$ mass spectrum, we Monte Carlo simulate events from this distribution for each viewing period. Since SagDEG has a large extent in the sky, we do not generate the photons from a point location but rather an ellipse with $a=4.0$ and $b=3.0$ centered at $l=5.0$ and $b=-14.0[2]$. We then spread these events as previously described and add in 
the effective area effects of the detector. We choose to scale the Monte Carlo counts based on the minimum $\chi^{2}$ value for the model as previously described. Figure 12 shows a plot of $\chi^{2}$ value as a function of this normalization factor.

We choose a value of 120 EGRET counts as our optimal normalization factor. FIGURE 13 \& FIGURE 14 show the longitude and latitude profiles for photon events generated by this extended source in the source region, with each color representing the same intermediate portions of the model as in the point source profiles.

\subsubsection{Variations of Extended Source Model}

We also choose to vary our extended source hypothesis by modeling differing WIMP masses, location of the center of the source and extent. We consider the location of our original extended source model as well as the location of 3EG J1847-3219 ( $l=3.21$ and $b=-13.37)$. Additionally, we consider the original extent of an ellipse with $a=4.0$ and $b=3.0$ and half-extent with $a=2.0$ and $b=1.5$.

We also consider the original extent and location with WIMP masses of $10 \mathrm{GeV}$ and $1000 \mathrm{GeV}$. Finally, we look at the scenario where the EGRET point source within the source region, 3EG J1847-3219, is considered part of the external (or in this case internal) point source contamination within the source region and the extended source accounts for the emission above the background model added to this contamination. The $\chi^{2}$ values associated with each of these varied models are located in TABLE 3.

\subsection{Hypothesis Testing}

We conduct $\chi^{2}$ testing for these different models. We use the standard comparison between the expected (model counts) values and those observed by EGRET (raw counts). For the case of the various extended source models, we choose to normalize in order to minimize the $\chi^{2}$ value, so these normalization factors present the most likely EGRET count values associated with these source models for best consistency with the data. 


\section{FUTURE ANALYSIS}

\subsection{Expansion of Analysis to GLAST}

We would like to explore the increased sensitivity GLAST will bring to high-energy astrophysics and specifically our analysis of extended source WIMP annihilation in SagDEG. In order to do this, we have decided to modify our previous method through the utilization of GLAST response functions.

Since no GLAST data has been taken, we cannot extract an apt background model from real data. Thus in order to run an analysis, which aims to simply highlight the improvement GLAST will offer, we plan to scale our current background model and EGRET count values to values appropriate for GLAST.

We will also model the various extended source models using the GLAST detector response functions. Since the LAT is more sensitive than EGRET, this will allow for a much more detailed analysis of this region.

After scaling the counts data (increasing count statistics decreases the statistical error), we will add the point source contamination within the source region and our new GLAST-treated extended source model and compare to the GLAST-scaled EGRET data.

\subsection{Extension to Different Energy Range}

We would like to increase the sensitivity of our analysis in the future by fine-tuning our analysis into different energy bands, which minimize the errors. In order to determine an optimal energy cut, we have conducted signal to background analysis for various energy cuts. We hope to split our analysis into two different energy bands, one which minimizes statistical error while the other minimizes systematic error. We took the WIMP annihilation spectra created by Pythia and integrated $\frac{d N}{d E}$ from the energy cut value to $\infty$ for each WIMP mass value. Utilizing the same cut values, we integrate a simplified function for the diffuse background. We use a power law spectrum with $\gamma=2.1[6]$. Finally, we plotted the signal to background ratio and signal to $\sqrt{\text { background }}$ ratio and selected the cuts, which will minimize the two previously mentioned errors.

We have selected the following two bins in which to conduct our analysis of the $100 \mathrm{GeV}$ mass WIMP: 1) $100 \mathrm{MeV}$ to $1 \mathrm{GeV}$ and 2) $1 \mathrm{GeV}$ to $10 \mathrm{GeV}$. The analysis will be repeated in these two 
bands in future work.

\section{RESULTS AND CONCLUSIONS}

We find that our method of extracting a background model from the data is both successful and simple. We were not forced to consider the varying portions of the diffuse background and thus posed with the challenge of adequately modeling these manifold contributions. In subtracting only point source bleeding, we were certain that we did not remove anything from the EGRET data that could be considered signal. Thus we truly created an upper bound of the background model, which allowed us to be sensitive to pure signal. Additionally, since the model was extracted from the viewing periods we chose to focus on, it is tailored specifically to our local analysis. The simplicity of our background model also allows for easy integration of the background with our source models.

However, since the background model is different from the EGRET diffuse model, we face the challenge of dealing with normalization values for our Monte Carlo simulations. The implementation of a different background model requires that the number of counts attributed to any source above the background will differ from the EGRET values (which are based on the diffuse value). Thus, our modeling of the point source and extended source hypotheses was limited to a selection of normalization factors depending on minimum $\chi^{2}$ values. This is simply one method to deal with this delicate issue.

TABLE 3 lists the $\chi^{2}$ values associated with each of the models we tested. We found that the various extended source models (with exception of the half-extent model at the original location) generate lower $\chi^{2}$ values than the no source or original point source values. The half-extent, pointsource located extended source has the lowest $\chi^{2}$ value, followed by the original extent and location with 3EG J1847-3219 as part of the background. We found that the $\chi^{2}$ values of the various extended source models (including those of the varying WIMP masses) are very similar to each other.

These results reflect our use of a $\chi^{2}$ minimization normalization process for the extended source models, while the point source model was constrained to the normalization calculated from the Third EGRET Catalog. When we allow the point source normalization to vary according to the corresponding minimum $\chi^{2}$ value, it becomes the lowest $\chi^{2}$ value, again highlighting the impact 
of this normalization process. However, this analysis indicates that an extended source could be responsible for the emission seen in this region and specifically a source corresponding to the extent and spectrum of WIMP clump located in the SagDEG fits the data within errors. This result pushes for further analysis of this region using methods mentioned above and hopefully GLAST data in the future.

Using 31 degrees of freedom (32 bins in our analysis with the normalization optimization process eliminating one), we find that we cannot reject either our point source or extended source models (we can only reject the model displaying no source in the region). Thus we have succeeded in corroborating EGRET's identification of 3EG 1847-3219 as a point source, using entirely different

methods. Additionally, our half-extent and point source located extended source's low $\chi^{2}$ value suggests that the dark matter distribution within SagDEG might be uneven, with most of the clumping in the area of the EGRET unidentified point source.

We hope to minimize systematic and statistical errors through the expansion of our analysis into two different energy bands. We think that this process will allow us to maximize our exploration of an extended source model since it's spectrum is much harder than that of the diffuse background model, thus analyzing the data in a higher energy band (e.g. $>1 \mathrm{GeV}$ ) will allow us to focus on pure signal in the source region.

We also hope to see the potential GLAST has for improving the sensitivity of this analysis. In our GLAST analysis, we plan on using a modified version of our background model, which was taken from EGRET data. We feel that this process will enable us to adequately show the capabilities of GLAST in the capacity of this analysis. However, with the possibility of obtaining a much more robust and intricate background model taken from the more sensitive GLAST data, we speculate that this analysis will provide more conclusive results with smaller error bars. We hope that this fact will allow GLAST to delve further into the exploration and understanding of dark matter.

\section{ACKNOWLEDGMENTS}

This research was conducted at the Stanford Linear Accelerator Center (SLAC). I would like to thank the U.S. Department of Energy, Office of Science, for providing me with the unique oppor- 
tunity of participating in the SULI program. I would especially like to thank my mentor Larry Wai for his enthusiasm, guidance and insight. I would also like to thank James Lindesay and Helen Quinn for providing a great experience and environment. Special thanks to Seth Digel, Jim Chiang and Reshmi Mukherjee for their EGRET expertise.

\section{References}

[1] Griest, K. 1996, Gateway Reviews, No. 2.

[2] Helmi, A. \& White, Simon D.M. 2001, MNRAS, 323 (529H).

[3] Baltz, E.A. \& Wai, L. 2004, Phys. Rev. D, 70 (023512).

[4] Hartman, R.C., et al. 1999, ApJS, 123 (79H).

[5] Sreekumar, P., et al. 1992, ApJ, 400 (67S).

[6] Hunter, S.D., et al. 1997, ApJ, 481 (205H).

[7] Ohishi M., Mori, M. \& Walker, M. 2002, Universal Academy Press, Inc. (1-8).

[8] Sjostrand, T., Eden, P., Friberg, L. Lonnblad, G. Miu, Mrenna, S. \& Norrbin, E. 2001, Computer Phys. Commun., 135 (238, LU TP 00-39, hep-ph/0010017)

[9] Combi, J.A., Romero, G.E., Benaglia, P. \& Jonas, J.L. 2001, A\&A, 336 (1047C)

[10] Grenier, I.A., Casandjian, J.M., \& Terrier, R. 2005, Science, 307 (1292).

[11] Jaffe, T.R., Bhattacharya, D., Dixon, D.D., \& Zych, A.D. 1997, ApJ, 484L (129J).

[12] Nolan, P.L., Tompkins, W.F., Grenier, I.A., \& Michelson, P.F. 2003, ApJ, 597 (615N).

[13] Peirani, S., Mohayaee, R., \& de Freitas Pacheco, J.A. 2004, Phys. Rev. D, 70 (3503P).

[14] Sreekumar, P., et al. 1998, ApJ, 494 (523S).

[15] Wai, L. 2005, NewAR, 49 (307W). 


\section{TABLES AND FIGURES}

\begin{tabular}{|c|c|c|c|c|c|}
\hline ViewingPeriod & Start & End & $l$ & $b$ & $3 E G J 1847-3219$ Counts \\
\hline 5.0 & 1991 Jul 12 & 1991 Jul 26 & 0.00 & -4.00 & $<67$ \\
\hline 209.0 & 1993 Feb 09 & 1993 Feb 22 & 0.24 & -34.01 & 35 \\
\hline 226.0 & 1993 Jun 19 & 1993 Jun 29 & 355.00 & 5.00 & $<34$ \\
\hline 232.0 & 1993 Aug 24 & 1993 Sep 07 & 347.50 & 0.00 & $<40$ \\
\hline 323.0 & 1994 Mar 22 & 1994 Apr 05 & 356.84 & -11.29 & $<50$ \\
\hline
\end{tabular}

Table 1: Viewing Periods: number, starting and ending dates, longitude and latitude of instrument z-axis, and number of counts associated with 3EG J1847-3219.

\begin{tabular}{|c|c|c|c|}
\hline Source & $l$ & $b$ & $\gamma$ \\
\hline $3 E G J 1736-2908$ & 358.79 & 1.56 & $2.18 \pm 0.12$ \\
$3 E G J 1744-3011$ & 358.85 & -0.52 & $2.17 \pm 0.08$ \\
$3 E G J 1746-2851$ & 0.11 & -0.04 & $1.70 \pm 0.07$ \\
$3 E G J 1800-3955$ & 352.05 & -8.24 & $3.10 \pm 0.35$ \\
$3 E G J 1834-2803$ & 5.92 & -8.97 & $2.62 \pm 0.20$ \\
$3 E G J 1850-2652$ & 8.58 & -11.75 & $2.29 \pm 0.45$ \\
$3 E G J 1858-2137$ & 14.21 & -11.15 & $3.45 \pm 0.38$ \\
$3 E G J 1911-2000$ & 17.03 & -13.29 & $2.39 \pm 0.18$ \\
\hline $3 E G J 1847-3219^{*}$ & 3.21 & -13.37 & 2.67 \\
\hline
\end{tabular}

Table 2: Parameters associated with point sources: EGRET source name, galactic longitude and latitude, and power-law index $\gamma$. ${ }^{*}$ Point Source 3EG J1847-3219 is located within source region.

\begin{tabular}{|c|c|c|c|c|c|c|}
\hline Model & $l$ & $b$ & $a$ & $b$ & OptimalNormalization & $\chi^{2}$ \\
\hline NoSource & - & - & - & - & - & 69.1256 \\
PointSource & 3.21 & -13.37 & - & - & - & 39.2362 \\
AlternativePointSource & 3.21 & -13.37 & - & - & 210 & 25.7097 \\
\hline Orig.Ext.Src. & 5.0 & -14.0 & 4.0 & 3.0 & 120 & 36.1757 \\
Orig.atNewLoc. & 3.21 & -13.37 & 4.0 & 3.0 & 130 & 34.0208 \\
Half - ExtentatOrig.Loc. & 5.0 & -14.0 & 2.0 & 1.5 & 50 & 44.0358 \\
Half-ExtentatNewLoc. & 3.21 & -13.37 & 2.0 & 1.5 & 100 & 28.3630 \\
\hline 10GeVmass & 5.0 & -14.0 & 4.0 & 3.0 & 130 & 36.7441 \\
1000GeVmass & 5.0 & -14.0 & 4.0 & 3.0 & 120 & 36.9587 \\
\hline Orig.withPSinBG & 5.0 & -14.0 & 4.0 & 3.0 & 80 & 32.2346 \\
Half-ExtentwithPSinBG & 5.0 & -14.0 & 2.0 & 1.5 & 60 & 35.8630 \\
\hline
\end{tabular}

Table 3: $\chi^{2}$ values associated with each model considered: Model, longitude and latitude, semimajor and semi-minor axis of extent, optimal normalization count value and $\chi^{2}$ value. 
EGRET AND GLAST: Angle of Incidence vs. Effective Area for $100 \mathrm{MeV}$

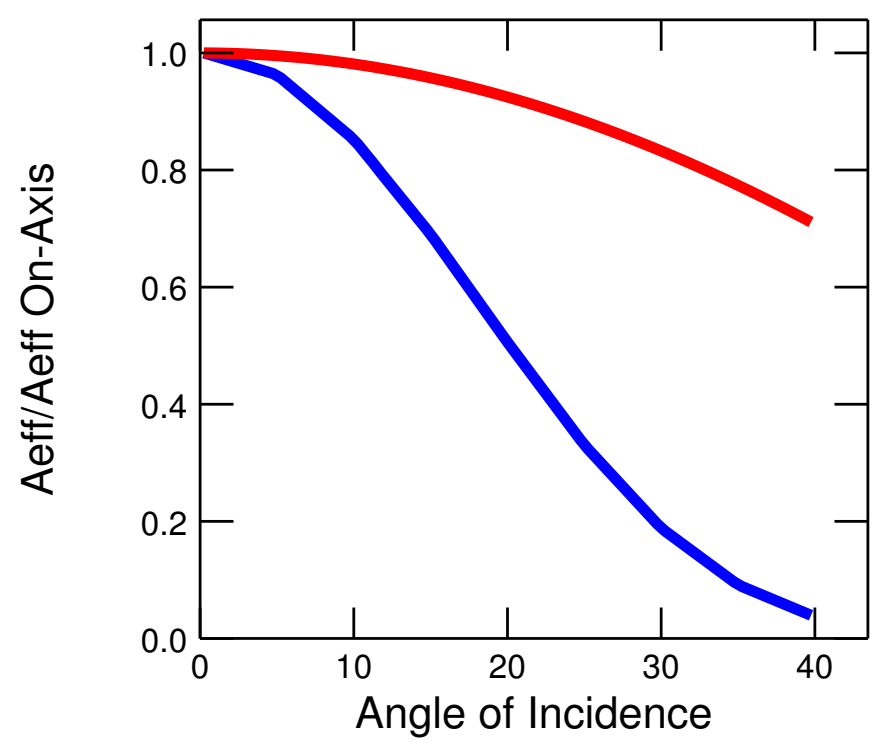

Figure 1: Effective area/effective area on-axis of EGRET (BLUE) and GLAST (RED) as a function of angle of incidence for energy of $100 \mathrm{MeV}$.

3EG J1850-2652 MC Spectrum of True Energies

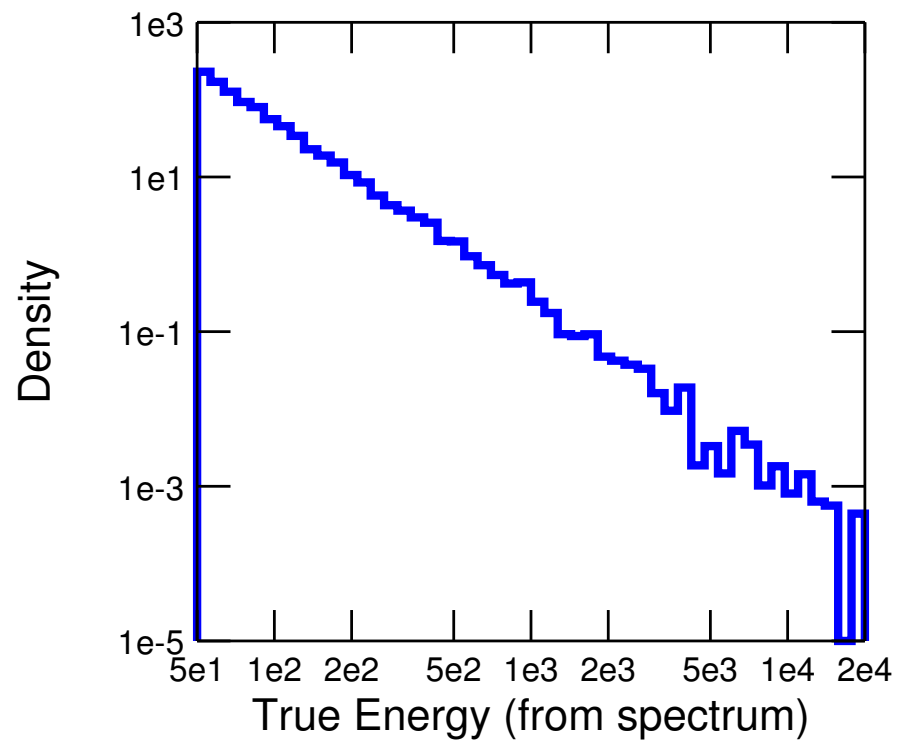

Figure 2: True Energy Spectrum of 3EG J1850-2652. (MEV) 
3EG J1850-2652 MC Spectrum of Energies Corrected for Dispersion

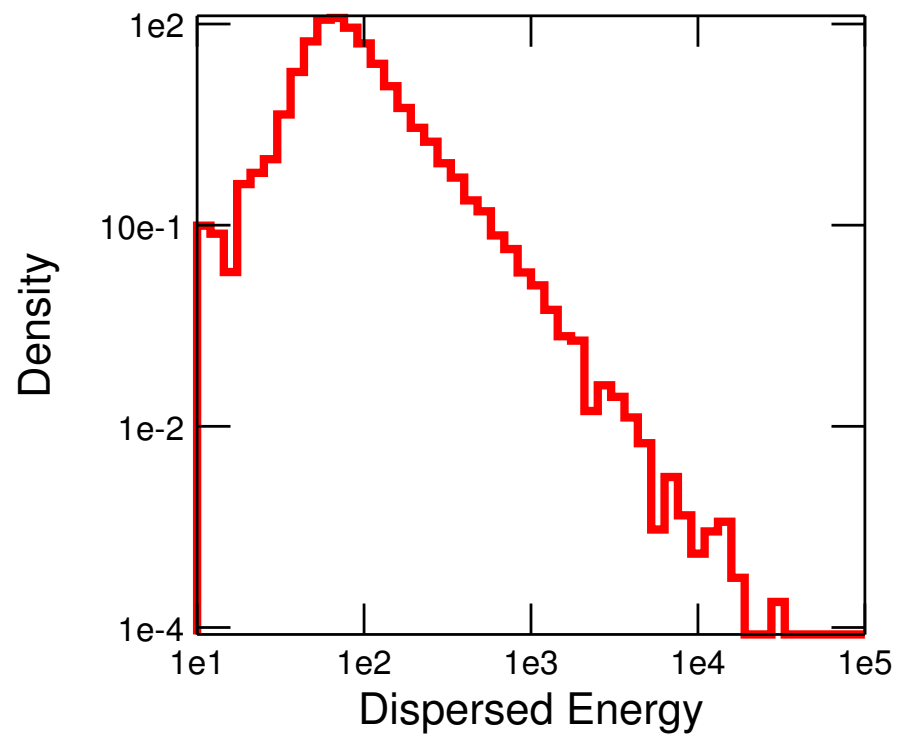

Figure 3: Energy spectrum after dispersion function employed on 3EG J1850-2652. (MEV)
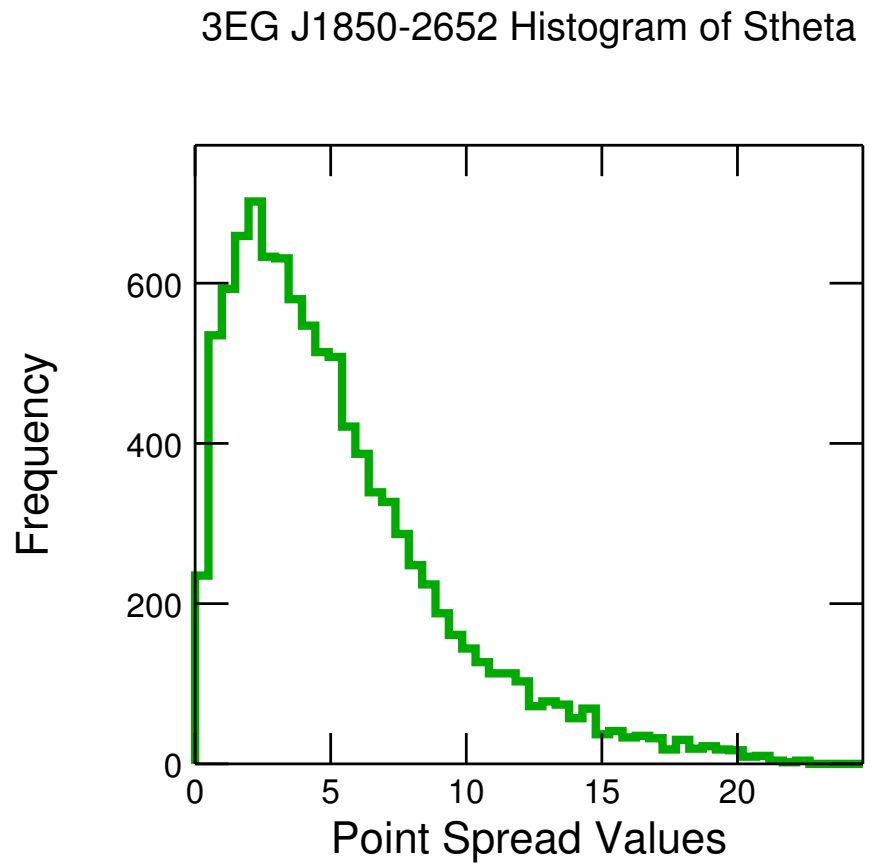

Figure 4: Counts as a function of angular distance (Point Spread Function) for 3EG J1850-2652. (Degrees) 


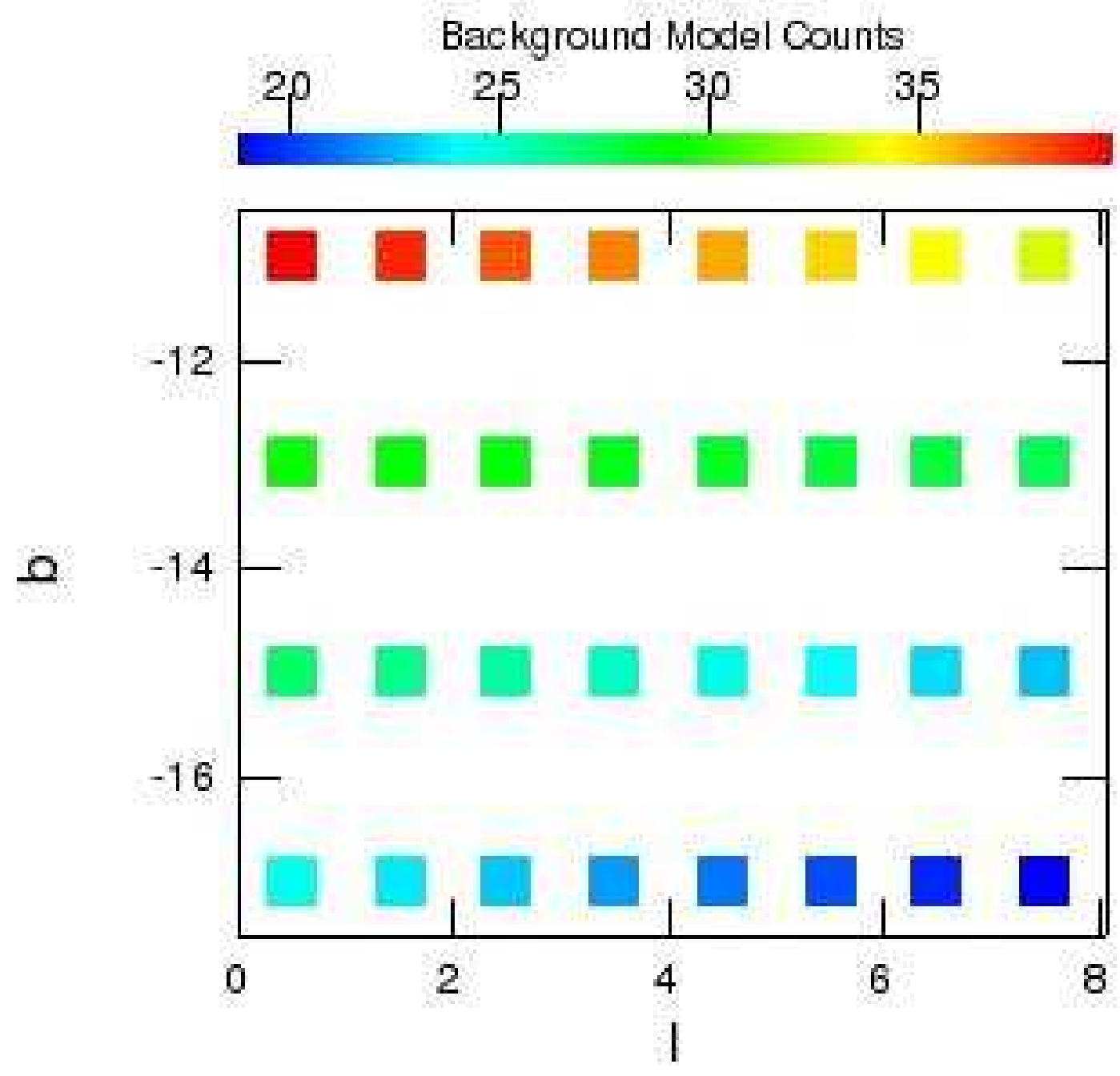

Figure 5: Three-dimensional background step-function model. 


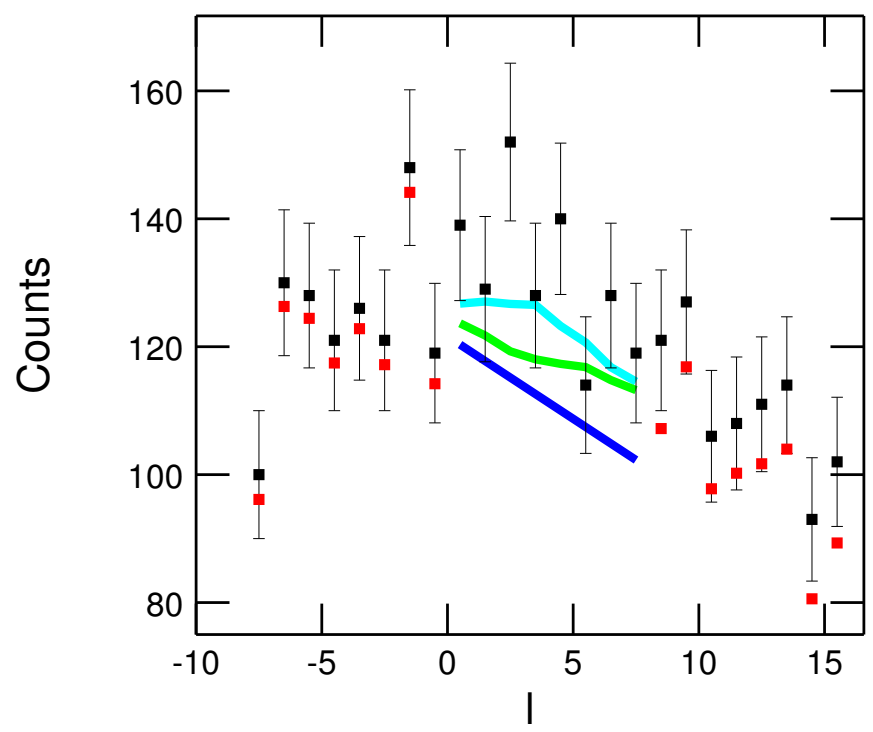

Figure 6: Longitude profile of point source emission. (Different colors explained in Section 3.2.1)

b x EGRET VP5 Counts (SOURCE REGION) - Point Source Model

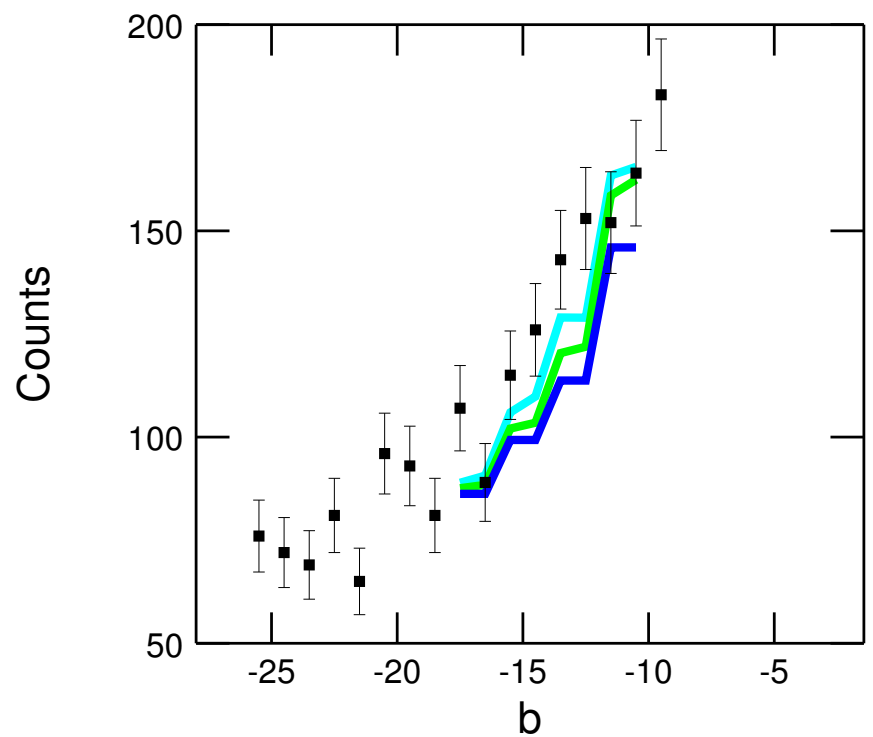

Figure 7: Latitude profile of point source emission. (Different colors explained in Section 3.2.1) 
Nomalization vs. Chi-2 Values (POINT SOURCE)

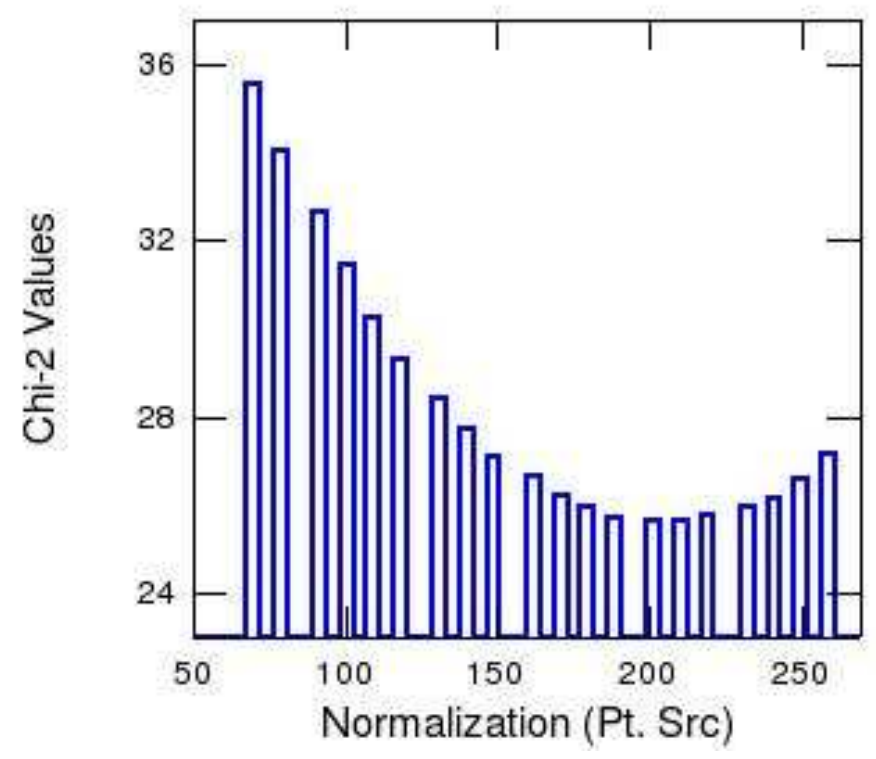

Figure 8: $\chi^{2}$ value as a function of scaling normalization value for point source model.

I $\times$ EGAET VP5 Count (SOUACE REGION) - Point Source Modd

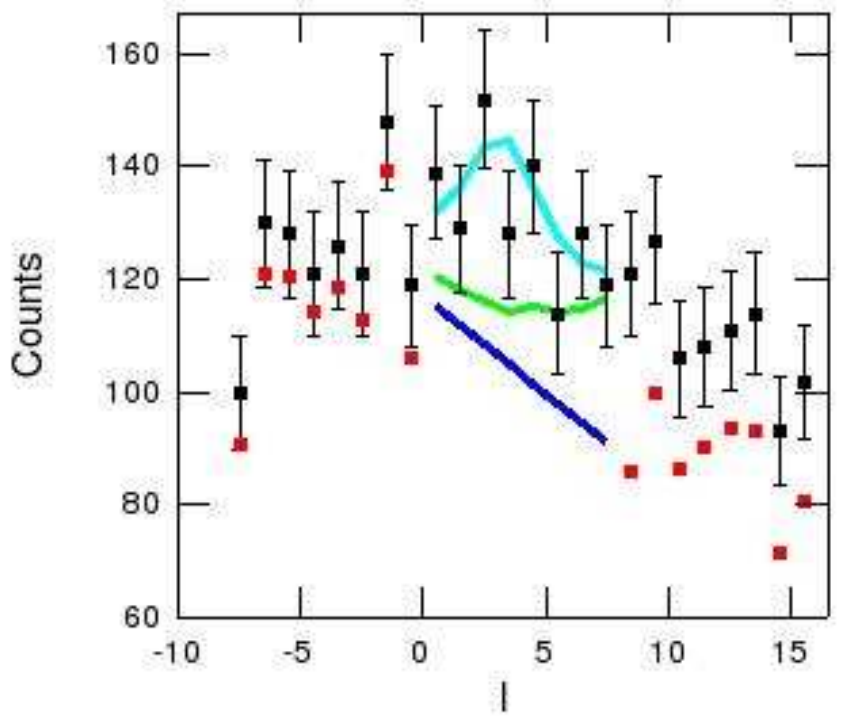

Figure 9: Longitude profile of alternate point source model. (Different colors explained in Section 3.2.1) 


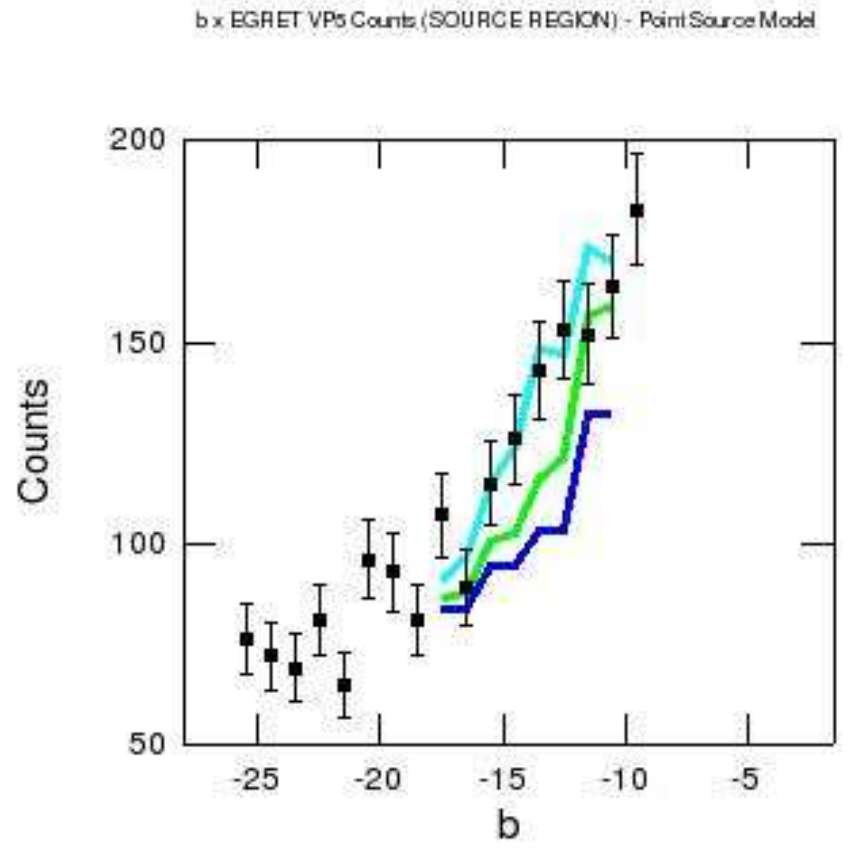

Figure 10: Latitude profile of alternate point source model. (Different colors explained in SEction 3.2.1)

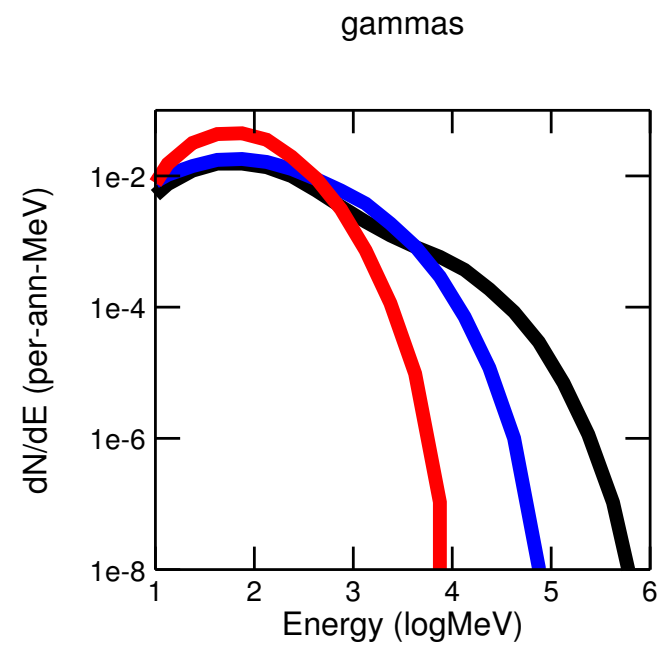

Figure 11: Energy spectra for WIMP annihilation for varying WIMP mass. red: $10 \mathrm{GeV}$, Blue: $100 \mathrm{GeV}$, and Black: $1000 \mathrm{Gev}$. [Plot generated by Larry Wai] 
Normalization vs. Chi-2 Values (EXTENDED SOURCE)

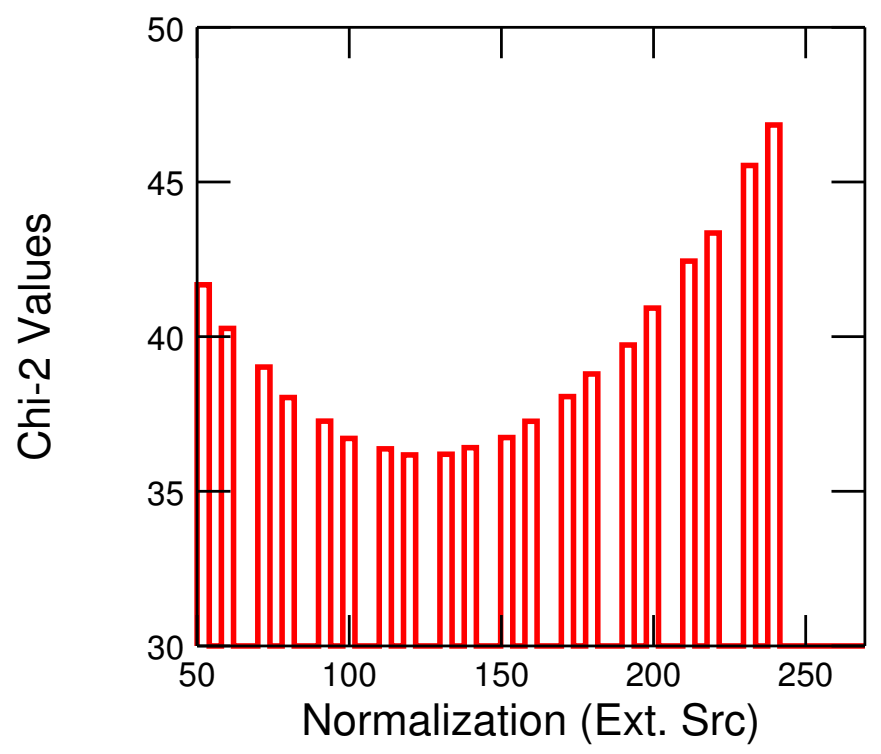

Figure 12: $\chi^{2}$ value as a function of scaling normalization value for extended source model.

Ix EGRET VP5 Counts (SOURCE REGION) - Extended Source Model

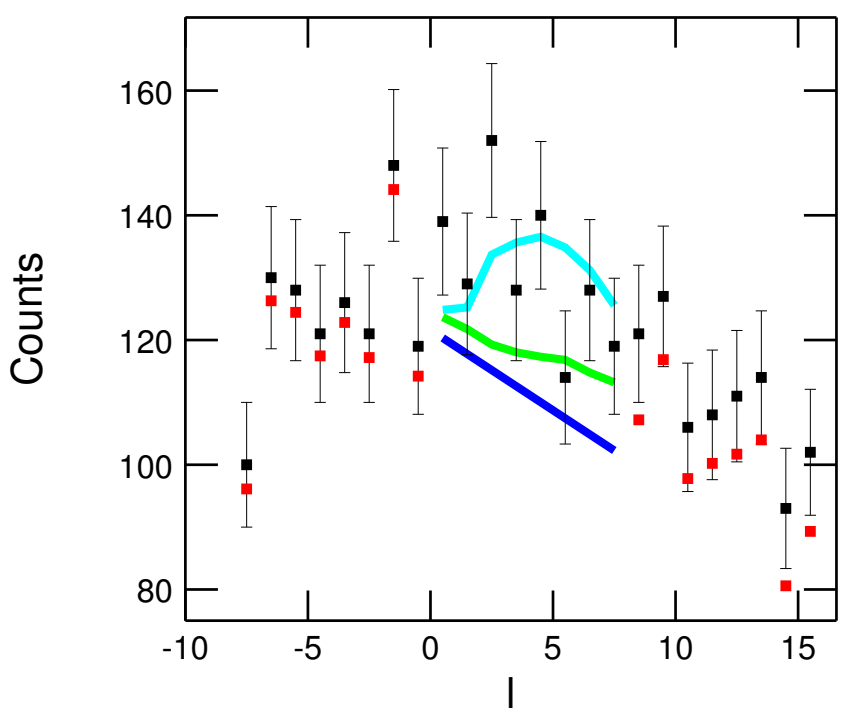

Figure 13: Longitude profile of extended source emission. (Different colors explained in Section 3.2.2) 
b x EGRET VP5 Counts (SOURCE REGION) - Extended Source Model

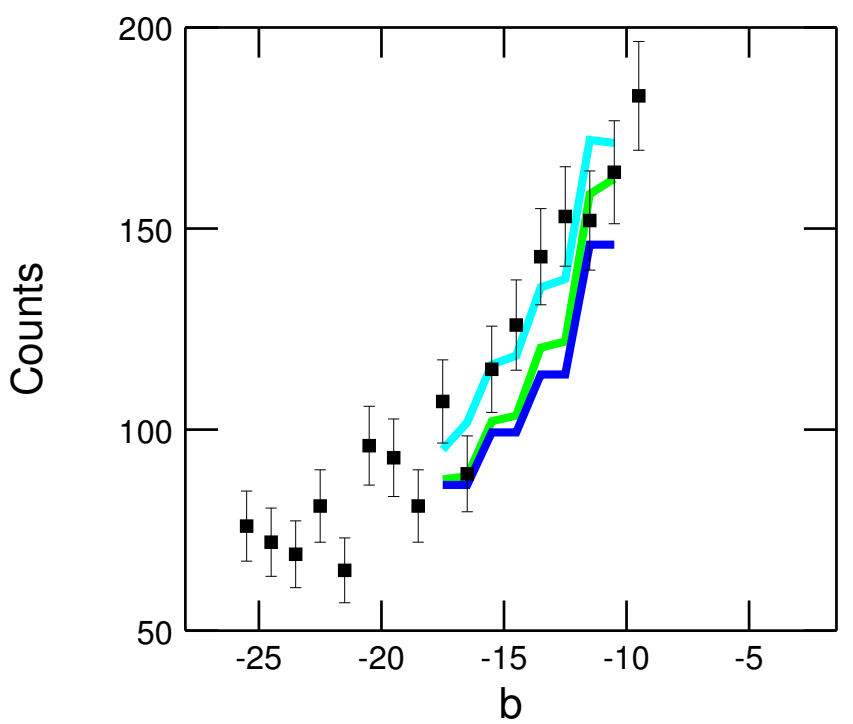

Figure 14: Latitude profile of extended source emission. (Different colors explained in Section 3.2.2) 